\title{
Percutaneous versus Surgical Revascularization for Acute Myocardial Infarction
}

\author{
Tariq Enezate ${ }^{1}$, Cliff Chen ${ }^{2}$, Kristina Gifft ${ }^{3}, \mathrm{Jad}_{\mathrm{O}} \mathrm{Omran}^{4}$, Mohammad Eniezat $^{5}$, and \\ Michael Readon ${ }^{6}$ \\ ${ }^{1}$ University of California Los Angeles \\ ${ }^{2}$ University of Missouri Health System \\ ${ }^{3}$ University of Missouri Columbia Health Care \\ ${ }^{4}$ University of California San Diego \\ ${ }^{5}$ Jordan University of Science and Technology \\ ${ }^{6}$ Houston Methodist Hospital
}

August 3, 2020

\begin{abstract}
Acute myocardial infarction (AMI) is a common medical condition that requires appropriate revascularization in a timely manner. Percutaneous revascularization (PR) was the first line treatment option when feasible. Limited data is available comparing PR to surgical revascularization (SR) in the AMI setting. Study population was extracted from the 2016 Nationwide Readmissions Data using International Classification of Diseases, tenth edition codes for AMI, PR, SR, and procedural complications. Endpoints included in-hospital all-cause mortality, length of index hospital stay (LOS), stroke, acute kidney injury, bleeding, blood transfusion, acute respiratory failure, and total hospital charges. The study identified 45,539 discharges with a principal admission diagnosis of AMI who had either PR or SR as a principal procedure. Single vessel revascularization was performed in $67.8 \%$ (93.1\% had PR versus $6.9 \%$ had SR, $\mathrm{p}<0.01)$. Multivessel revascularization was performed in $32.2 \%(64.8 \%$ had PR versus $35.2 \%$ had $\mathrm{SR}, \mathrm{p}<0.01)$. In comparison to $\mathrm{SR}, \mathrm{PR}$ was associated with higher in-hospital all-cause mortality $(\mathrm{P}<0.01)$, shorter LOS $(p<0.01)$, and lower incidence of post-procedural stroke $(\mathrm{p}<0.01)$, acute kidney injury $(\mathrm{p}<0.01)$, bleeding $(\mathrm{p}<0.01)$, need for blood transfusion $(\mathrm{p}<0.01)$, acute respiratory failure $(\mathrm{p}<0.01)$, and total hospital charges $(\mathrm{p}<0.01)$. In a subgroup analysis, SR mortality benefit persisted in patients who had multivessel revascularization, but not in single vessel revascularization. In patients presented with AMI, PR was associated with higher in-hospital all-cause mortality but lower morbidity, shorter LOS, and lower total hospital charges than SR. However, the mortality benefit of SR was seen in multivessel revascularization only, and not in single vessel revascularization.
\end{abstract}

\section{Percutaneous versus Surgical Revascularization for Acute Myocardial Infarction}

Running Title: PCI vs CABG for AMI

Authors: Tariq Enezate MD ${ }^{\mathrm{a}}$, Cliff Chen $\mathrm{MD}^{\mathrm{b}}$, Kristina Gifft DO ${ }^{\mathrm{b}}$, Jad Omran MD ${ }^{\mathrm{c}}$, Mohammeid Eniezat $\mathrm{MS}^{\mathrm{d}}$, Michael Readon $\mathrm{MD}^{\mathrm{e}}$

\section{Affiliations:}

${ }^{a}$ Division of Cardiology, UCLA-Harbor Medical Center, Los Angeles, CA

b Department of Internal Medicine, University of Missouri Health Care, Columbia, MO

${ }^{c}$ University of California, San Diego, CA 
d Jordan University of Science and Technology,

e Houston Methodist Hospital, Houston, TX

Presentations: CRT, February 2020; Washington DC

Category: Acute coronary syndrome, Cardiac catheterization/intervention, CABG, Myocardial infarction, Outcomes

Word Count: 4060

Corresponding Author: Kristina K Gifft DO 1 Hospital Drive, University of Missouri - Columbia; Dept of Internal Medicine

Columbia MO, 65212 Email: gifftk@health.missouri.edu, Cell: 607-351-1951

\section{ABSTRACT}

\section{Introduction}

Acute myocardial infarction (AMI) is a common medical condition in our clinical practice that should be treated with appropriate revascularization in a timely manner. Percutaneous revascularization (PR) has been the first line treatment option when feasible. Limited data is available comparing PR to surgical revascularization (SR) in the AMI setting.

\section{Methods}

Study population was extracted from the 2016 Nationwide Readmissions Data using International Classification of Diseases, tenth edition, clinical modifications/procedure coding system codes for AMI, PR, SR, and procedural complications. Study endpoints included in-hospital all-cause mortality, length of index hospital stay (LOS), stroke, acute kidney injury, bleeding, need for blood transfusion, acute respiratory failure, and total hospital charges.

\section{Results}

The study identified 45,539 discharges with a principal admission diagnosis of AMI (38.7\% ST elevation and $61.3 \%$ non-ST elevation) who had either PR or SR as a principal procedure $(79.1 \%$ PR versus $20.9 \%$ $\mathrm{SR}$ ). Single vessel revascularization was performed in $67.8 \%$ (93.1\% had PR versus $6.9 \%$ had $\mathrm{SR}, \mathrm{p}<0.01)$. Multivessel revascularization was performed in $32.2 \%$ (64.8\% had PR versus $35.2 \%$ had SR, $\mathrm{p}<0.01)$. $83 \%$ of SR was in the setting of non-ST elevation AMI (NSTEMI). In comparison to SR, PR was associated with higher in-hospital all-cause mortality $(3.7 \%$ versus $2.2 \%, \mathrm{P}<0.01)$, shorter LOS (4.3 versus 11.6 days, $\mathrm{p}<0.01)$, and lower incidence of post-procedural stroke $(1.0 \%$ versus $1.8 \%, \mathrm{p}<0.01)$, acute kidney injury ( $14.9 \%$ versus $24.8 \%, \mathrm{p}<0.01$ ), bleeding ( $4.3 \%$ versus $47.1 \%, \mathrm{p}<0.01)$, need for blood transfusion $(2.9 \%$ versus $18.5 \%, \mathrm{p}<0.01)$, acute respiratory failure ( $10.7 \%$ versus $19.8 \%, \mathrm{p}<0.01)$, and total hospital charges $(120,590 \$$ versus $229,917 \$, \mathrm{p}<0.01)$. These results persist after adjustment for baseline characteristics. In a subgroup analysis, SR mortality benefit persisted in patients who had multivessel revascularization (in both ST and non-ST elevation AMI), but not in single vessel revascularization.

\section{Conclusions}

In patients presented with AMI, PR was associated with higher in-hospital all-cause mortality but lower morbidity, shorter LOS, and lower total hospital charges than SR. However, the mortality benefit of SR was seen in multivessel revascularization only, and not in single vessel revascularization.

\section{INTRODUCTION}

Coronary artery disease (CAD) affects over 18 million people in the United States, with over 8.4 million cases present as acute myocardial infarctions. ${ }^{[1]}$ Half of acute myocardial infarctions (AMI) are associated with multivessel disease. ${ }^{[2]}$ Data suggest better outcomes with complete percutaneous revascularization (PR) vs culprit-only PR in the setting of multivessel AMI mainly driven by less need for future urgent 
revascularization. ${ }^{[2,3]}$ Incomplete PR in patients with multivessel CAD, has been recognized as an independent predictor of mortality ${ }^{[4,5]}$. On the other hand, surgical revascularization (SR) is the preferred strategy over PR in stable coronary disease when there is complex anatomy, diabetes, and/or left ventricular dysfunction ${ }^{[6-10]}$. Moreover, SR allows for more complete revascularization than PR and less need for future revascularization. ${ }^{[7][11]}$

Some studies have shown PR to be associated with lower rates of early stroke than SR, and is known globally to be more widely available. ${ }^{[8]} \mathrm{PR}$ can achieve revascularization of the culprit vessel in a more timely manner which is of particular importance in the setting of AMI. ${ }^{[12-15]}$ There are limited data comparing the two revascularization strategies in the setting of AMI given to pros and cons of each strategy.

\section{METHODS}

\section{Data Source}

The Nationwide Readmissions Data (NRD) is a part of the Healthcare Cost and Utilization Project (HCUP) databases, which has been developed through a Federal-State-Industry partnership and sponsored by the Agency for Healthcare Research and Quality (AHRQ). HCUP databases include the largest collection of de-identified longitudinal hospital care data in the United States, with safeguards to protect the privacy of individual patients, physicians, and hospitals. The NRD contains more than a hundred clinical and nonclinical variables for each hospital stay including a verified patient linkage number for linking hospital visits for the same patient across hospitals, International Classification of Diseases, Tenth Revision, Clinical Modification/Procedure Coding System (ICD-10-CM/PCS) for principal and secondary procedures and diagnoses (including comorbidities and complications), age, gender, length of stay (LOS), and others. ${ }^{[16,17]}$

\section{Study Cohort}

ICD-10-CM/PCS codes were used to search discharges in the 2016 NRD who had a principle diagnosis of AMI (including ST and non-ST elevation AMIs) and a principle procedure of either PR or SR during the index hospitalization; baseline characteristics, comorbidities, in-hospital procedural complications, and endpoints of interest were subsequently extracted. To differentiate post-procedural complications from chronic conditions, the 2016 NRD has a present-on-admission indicator for chronic conditions that present on admission. We also utilized the ICD-10-CM codes used in the Elixhauser comorbidity index to identify comorbid conditions and utilized ICD-10-CM codes that are specific for post-procedural complications, Supplemental Table 1.[17] We excluded patients that did not have AMI as a principle diagnosis and AMIs that were not treated invasively (i.e. PR or SR) or when the revascularization was not the principle procedure. We also excluded AMIs that were treated with a combination of both PR and SR during the same indexed admission. The NRD excludes discharges with missing age, missing or questionable linkage numbers or from hospitals with more than $50 \%$ of their discharges excluded because of these criteria, as patients treated in these hospitals may not be reliably tracked over time. ${ }^{[16]}$ All HCUP recommendations and best practices to use the HCUP datasets highlighted by Khera et al were followed. ${ }^{[18]}$

\section{Study Endpoints}

The study endpoints included in-hospital all-cause mortality, LOS, post-procedural stroke, acute kidney injury, bleeding, need for blood transfusion, acute respiratory failure, and total hospital charges. The 2016 NRD reports in-hospital all-cause deaths and mean LOS. The other endpoints were assessed during the index hospitalization using specific ICD-10 codes for post-procedural complications, Supplemental Table 1. Stroke included new intra or post-procedural cerebral infarction secondary to bleeding, thrombosis and/or embolism to one or more cerebral arteries. Acute kidney injury included any new post-procedural acute worsening of kidney function. Bleeding included any circulatory or central nervous system bleeding during or post-procedure, or post-procedure hemorrhage/anemia. Transfusion included blood or blood product transfusion post-procedure. Acute respiratory failure included new post-procedural hypoxemic or hypercapnic respiratory failure or acute worsening of chronic respiratory failure. Total hospital charges represented how much the hospital billed for the service but not necessarily the actual cost or the amount the hospital 
actually received.

\section{Statistical Analysis}

Statistical Analysis System (SAS) software 9.4 (TS1M4, SAS Institute Inc, Cary, North Carolina) was used for data extraction and statistical analysis which was performed on unweighted (i.e. actual number) discharges. Pearson's Chi-Square of Independence and unpaired-samplet -test were used to compare the endpoints of interest and baseline characteristics in both PR and SR groups. Logistic regression was used to create propensity score, based on the basic demographics and baseline characteristics for one-to-one parallel, balanced propensity score matching model using a caliper of 0.001 . The McNemar test was used to compare paired categorical variables of the baseline characteristics and endpoints of interest, while paired-samples $t$-test was used to compare continuous variables. A two-tailed p-value of $<0.05$ was used for statistical significance. ${ }^{[19,20]}$

\section{RESULTS}

The 2016 NRD database included approximately 17.2 million discharges. There were 328,570 discharges with a principal diagnosis of AMI. Of which, 45,539 (38.7\% ST elevation and $61.3 \%$ non-ST elevation) had either PR $79.1 \%$ or SR $20.9 \%$ as a principal procedure, Figure 1. The mean age of the overall cohort was $64.8+$ 12.2 years and $29.8 \%$ were women. History of CAD, hypertension, hyperlipidemia, diabetes, and smoking were the most common comorbidities, Table 1.

SR group had significantly more comorbidities including congestive heart failure and diabetes. Single vessel revascularization was performed in $67.8 \%(93.1 \% \mathrm{PR}$ versus $6.9 \% \mathrm{SR}, \mathrm{p}<0.01)$. Multivessel revascularization was performed in $32.2 \%$ (64.8\% PR versus $35.2 \% \mathrm{SR}, \mathrm{p}<0.01) .16 .5 \%$ in the SR group had ST elevation AMI and $83.2 \%$ had non-ST elevations, while $44.6 \%$ of the PR group had ST elevation and $54.9 \%$ had non-ST elevation AMI, Table 1.

In comparison to SR, PR was associated with higher in-hospital all-cause mortality $(3.7 \%$ versus $2.2 \%$, $\mathrm{P}<0.01)$, shorter LOS (4.3 versus 11.6 days, $\mathrm{p}<0.01)$, and lower incidence of post-procedural stroke $(1.0 \%$ versus $1.8 \%, \mathrm{p}<0.01)$, acute kidney injury $(14.9 \%$ versus $24.8 \%, \mathrm{p}<0.01)$, bleeding $(4.3 \%$ versus $47.1 \%$, $\mathrm{p}<0.01)$, need for blood transfusion $(2.9 \%$ versus $18.5 \%, \mathrm{p}<0.01)$, acute respiratory failure $(10.7 \%$ versus $19.8 \%, \mathrm{p}<0.01)$, and total hospital charges $(120,590 \$$ versus $229,917 \$, \mathrm{p}<0.01)$ (Table 2$)$.

There were 6,938 comparable pairs were identified using propensity matching (Table 1), these pairs also comparable in terms of cardiogenic shock $(10.0 \%$ versus $9.5 \%, \mathrm{p}=0.35)$ and mechanical complications of AMI $(0.2 \%$ versus $0.2 \%, \mathrm{p}=055)$. The results remained consistent after propensity matching (Table 2 ).

In subgroup analysis, SR mortality benefit persisted in patients who had multivessel revascularization (in both ST and non-ST elevation AMI), but not in single vessel revascularization. Furthermore, the mortality benefit persisted in patients with and without diabetes, systolic heart failure, cardiogenic shock and mechanical complications of AMI.

\section{DISCUSSION}

In this study, SR of AMI was associated with lower in-hospital mortality but higher morbidity, longer LOS, and total hospital charges than PR. These results remained consistent in patients who had multiple vessel revascularization (in both ST and non-ST elevation AMI), but not single vessel, and in patients with/without diabetes, systolic heart failure, cardiogenic shock or mechanical complications of AMI, despite the fact that SR group had higher baseline comorbidities. These results persist after adjustment for baseline characteristics using propensity matching.

Most of the previous studies that showed comparable or superior outcomes of SR in patients with stable CAD, rather than AMI, and over a longer period of time. ${ }^{[21]}$ A pooled data from multiple trials that compared SR to PR in patients with NSTEMI showed that SR was associated with lower composite end points over 5 years which was mainly driven by lower infarction rather than lower mortality. ${ }^{[23]}$ However, another study showed a trend to lower events rate and lower mortality in comparison to PR. ${ }^{[24]}$ 
Advancements in surgical techniques such as off pump surgery, clampless and no-touch surgery, epiaortic ultrasonography, and minimally invasive/robotic SR all have contributed to lower both operative and longterm mortality and complications rates. ${ }^{[25]}$ Moreover, the heart-team approach for SR patient selection using a multidisciplinary team could increase the operative safety and success rate. ${ }^{[26]}$

Conduit selection has also showed to improve outcomes and mortality. ${ }^{[1]}$ Internal mammary artery (IMA) grafting to left anterior descending is a major survival determinant independently from the presence of other graft. This is likely because of superior patency rates in comparison to vein grafts ${ }^{[2]}$ and high proportion of elastic composition compared to muscle or adventitia making it more able to tolerate coronary blood flow. Furthermore, IMA grafts have physiological functions that result in anti-atherosclerotic effects by producing much greater levels of nitric oxide and decreased release of vasoconstrictors. Nitric oxide is a known potent angiogenic agent which initiates neocapillary and microvascular bed formation in the affected and adjacent areas. ${ }^{[1,27]}$ These factors could have resulted in the lower early in-hospital mortality seen in this study despite the higher complications rates.

Most of AMI results from non-flow-limiting lesions; however, PR treats flow-limiting lesions only therefore it is not expected to prevent new infarcts and subsequently lacks mortality benefit. On the other hand, SR bypasses the whole diseased segments which creates "surgical collateralizations"; a condition that allows revascularization of the diseased-vessel which subsequently causes no or nonfatal AMI which could also decrease mortality. ${ }^{[21]}$

This study is based on a large nation-wide database and represents real-world outcomes in the United States. It adds to the current literature that SR in the AMI setting is still a feasible option, especially when PR is expected to be suboptimal or results in incomplete revascularization, and might be associated with lower in-hospital mortality in patients with multivessel CAD.

\section{Limitations}

This was a retrospective study. ICD-10 codes do not specify the involved coronary artery, the location, severity, or complexity of the coronary lesions. The type and the intensity of the medical therapy, core laboratory and the exact ejection fraction were not provided. The details of the procedures, such doorto-intervention time, duration of the procedure, etc. could not be assessed. Furthermore, the reason why patients underwent SR vs PR could be determined. Long term outcomes could not be assessed.

\section{CONCLUSIONS}

Percutaneous revascularization was associated with higher in-hospital all-cause mortality but lower morbidity, shorter LOS, and lower total hospital charges than SR in patients with acute myocardial infarction. The mortality benefit of SR was seen in multivessel revascularization only, and not in single vessel revascularization.

\section{Disclosures}

All authors have no conflict of interest, financial disclosures, or relationship with industry.

\section{Acknowledgement}

None.

\section{REFERENCES}

[1] S.S. Virani, A. Alonso, E.J. Benjamin, et al, Heart Disease and Stroke Statistics2020 Update: A Report From the American Heart Association, Circulation. 141 (2020). https://doi.org/10.1161/CIR.0000000000000757.

${ }^{[2]}$ D.S. Wald, J.K. Morris, N.J. Wald, et al, Randomized Trial of Preventive Angioplasty in Myocardial Infarction, N. Engl. J. Med. 369 (2013) 1115-1123. https://doi.org/10.1056/NEJMoa1305520. 
${ }^{[3]} \mathrm{H}$. Xu, X. Zhang, J. Li, et al, Complete versus culprit-only revascularization in patients with ST-segment elevation myocardial infarction and multivessel disease: a meta-analysis of randomized trials, BMC Cardiovasc. Disord. 19 (2019) 91. https://doi.org/10.1186/s12872-019-1073-8.

[4] C. Wu, A.-M. Dyer, S.B. King, et al, Impact of Incomplete Revascularization on LongTerm Mortality After Coronary Stenting, Circ. Cardiovasc. Interv. 4 (2011) 413-421. https://doi.org/10.1161/CIRCINTERVENTIONS.111.963058.

${ }^{[5]}$ Z. Gao, B. Xu, Y. Yang, et al, Long-term outcomes of complete versus incomplete revascularization after drug-eluting stent implantation in patients with multivessel coronary disease, Catheter. Cardiovasc. Interv. 82 (2013) 343-349. https://doi.org/10.1002/ccd.24799.

[6] B.R. Chaitman, A.D. Rosen, D.O. Williams, et al, Myocardial Infarction and Cardiac Mortality in the Bypass Angioplasty Revascularization Investigation (BARI) Randomized Trial, Circulation. 96 (1997) 21622170. https://doi.org/10.1161/01.CIR.96.7.2162.

${ }^{[7]}$ P.W. Serruys, M.-C. Morice, A.P. Kappetein, et al, Percutaneous Coronary Intervention versus CoronaryArtery Bypass Grafting for Severe Coronary Artery Disease, N. Engl. J. Med. 360 (2009) 961-972. https://doi.org/10.1056/NEJMoa0804626.

${ }^{[8]}$ M.E. Farkouh, M. Domanski, L.A. Sleeper, et al, Strategies for Multivessel Revascularization in Patients with Diabetes, N. Engl. J. Med. 367 (2012) 2375-2384. https://doi.org/10.1056/NEJMoa1211585.

${ }^{[9]}$ E.J. Velazquez, K.L. Lee, R.H. Jones, et al, Coronary-Artery Bypass Surgery in Patients with Ischemic Cardiomyopathy, N. Engl. J. Med. 374 (2016) 1511-1520. https://doi.org/10.1056/NEJMoa1602001.

${ }^{[10]}$ M.R. Patel, J.H. Calhoon, G.J. Dehmer, et al, ACC/AATS/AHA/ASE/ASNC/SCAI/SCCT/STS 2017 Appropriate Use Criteria for Coronary Revascularization in Patients With Stable Ischemic Heart Disease, J. Am. Coll. Cardiol. 69 (2017) 2212-2241. https://doi.org/10.1016/j.jacc.2017.02.001.

[11] C. Spadaccio, U. Benedetto, Coronary artery bypass grafting (CABG) vs. percutaneous coronary intervention (PCI) in the treatment of multivessel coronary disease: quo vadis? - a review of the evidences on coronary artery disease, Ann. Cardiothorac. Surg. 7 (2018) 506-515. https://doi.org/10.21037/acs.2018.05.17.

${ }^{[12]}$ P.T. O'Gara, F.G. Kushner, D.D. Ascheim, et al, 2013 ACCF/AHA Guideline for the Management of STElevation Myocardial Infarction, Circulation. 127 (2013). https://doi.org/10.1161/CIR.0b013e3182742cf6.

${ }^{[13]}$ L. Lambert, Association Between Timeliness of Reperfusion Therapy and Clinical Outcomes in STElevation Myocardial Infarction, JAMA. 303 (2010) 2148. https://doi.org/10.1001/jama.2010.712.

${ }^{[14]}$ B.R. Brodie, T.D. Stuckey, T.C. Wall, et al, Importance of time to reperfusion for 30-day and late survival and recovery of left ventricular function after primary angioplasty for acute myocardial infarction, J. Am. Coll. Cardiol. 32 (1998) 1312-1319. https://doi.org/10.1016/S0735-1097(98)00395-7.

[15] C.P. Cannon, Relationship of Symptom-Onset-to-Balloon Time and Door-to-Balloon Time With Mortality in Patients Undergoing Angioplasty for Acute Myocardial Infarction, JAMA. 283 (2000) 2941. https://doi.org/10.1001/jama.283.22.2941.

[16] 2014 Introduction to the NRD. Healthcare Cost and Utilization Project (HCUP), Agency Healthc. Res. Qual. Rockv. M. (n.d.). www. hcup-us. ahrq. gov/db/nation/nrd/NRD_Introduction_2014. jsp.

${ }^{[17]}$ K.L.C. Gibson T, Casto A, Young J, Impact of ICD-10- CM/PCS on Research Using Administrative Databases, . . HCUP Methods Ser. Rep. \# 2016- 02 ONLINE. July 25, 2016. U.S. Agency Healthc. Res. Qual. (n.d.). available: http://www.hcup-us.ahrq.gov/reports/methods/methods.jsp.

${ }^{[18]}$ R. Khera, S. Angraal, T. Couch, et al, Adherence to Methodological Standards in Research Using the National Inpatient Sample, JAMA. 318 (2017) 2011. https://doi.org/10.1001/jama.2017.17653. 
[19] M.A. Pourhoseingholi, A.R. Baghestani, M. Vahedi, How to control confounding effects by statistical analysis., Gastroenterol. Hepatol. from Bed to Bench. 5 (2012) 79-83. http://www.ncbi.nlm.nih.gov/pubmed/24834204.

${ }^{[20]}$ M.S.H.P. 2014. McDonald JH. Handbook of Biological Statistics. 3rd ed. Baltimore, No Title, (n.d.).

${ }^{21]}$ T. Doenst, A. Haverich, P. Serruys, et al, PCI and CABG for Treating Stable Coronary Artery Disease, J. Am. Coll. Cardiol. 73 (2019) 964-976. https://doi.org/10.1016/j.jacc.2018.11.053.

[22] S.-J. Park, J.-M. Ahn, Y.-H. Kim, et al, BEST Trial Investigators, Trial of everolimuseluting stents or bypass surgery for coronary disease., N. Engl. J. Med. 372 (2015) 1204-12. https://doi.org/10.1056/NEJMoa1415447.

${ }^{[23]}$ M. Chang, C.W. Lee, J.-M. Ahn, et al, Comparison of Outcome of Coronary Artery Bypass Grafting Versus Drug-Eluting Stent Implantation for Non-ST-Elevation Acute Coronary Syndrome., Am. J. Cardiol. 120 (2017) 380-386. https://doi.org/10.1016/j.amjcard.2017.04.038.

${ }^{[24]}$ P. Freitas, M. Madeira, L. Raposo, et al, Coronary Artery Bypass Grafting Versus Percutaneous Coronary Intervention in Patients With Non-ST-Elevation Myocardial Infarction and Left Main or Multivessel Coronary Disease., Am. J. Cardiol. 123 (2019) 717-724. https://doi.org/10.1016/j.amjcard.2018.11.052.

[25] S.J. Head, M. Milojevic, D.P. Taggart, et al, Current Practice of State-ofthe-Art Surgical Coronary Revascularization, Circulation. $136 \quad$ (2017) 1331-1345. https://doi.org/10.1161/CIRCULATIONAHA.116.022572.

[26] S.J. Head, S. Kaul, M.J. Mack, et al, The rationale for Heart Team decision-making for patients with stable, complex coronary artery disease, Eur. Heart J. 34 (2013) 2510-2518. https://doi.org/10.1093/eurheartj/eht059.

${ }^{[27]}$ D.P. Taggart, R. D'Amico, D.G. Altman, Effect of arterial revascularisation on survival: a systematic review of studies comparing bilateral and single internal mammary arteries., Lancet (London, England). 358 (2001) 870-5. https://doi.org/10.1016/S0140-6736(01)06069-X.

\section{Figure Legend}

Table 1: Demographics, baseline characteristics and comorbidities of percutaneous revascularization (PR) and surgical revascularization (SR) groups before and after propensity matching.

Table 2: Study endpoints before and after propensity matching.

Figure 1: Flowchart demonstrating data extraction process.

Supplemental Table 1: International Classification of Diseases, Tenth Revision, Clinical Modification/Procedure Coding System (ICD-10-CM/PCS) codes used to define baseline characteristics, comorbidities, and in-hospital and post-procedural complications from the 2016 Nationwide Readmissions Data (NRD).

Table 1: Demographics, baseline characteristics and comorbidities of percutaneous revascularization (PR) and surgical revascularization (SR) groups before and after propensity matching.

\begin{tabular}{lllllll}
\hline & Before Matching & Before Matching & & After Matching & After Matching & \\
& PR & SR & P-value & PR & SR & P-val \\
Number of patients & 35,989 & 9,550 & - & 6,938 & 6,938 & - \\
Mean Age in years (SD) & $64.7(12.6)$ & $65.1(10.7)$ & $<0.01$ & $65.9(12.5)$ & $65.3(10.7)$ & \\
Female & $30.8 \%$ & $25.7 \%$ & $<0.01$ & $31.0 \%$ & $28.1 \%$ & $<0.01$ \\
ST Elevation AMI & $44.6 \%$ & $16.5 \%$ & $<0.01$ & $21.7 \%$ & $21.5 \%$ & 0.81 \\
Non-ST Elevation AMI & $54.9 \%$ & $83.2 \%$ & $<0.01$ & $77.8 \%$ & $78.0 \%$ & 0.78 \\
Single-vessel Revascularization & $79.9 \%$ & $22.2 \%$ & $<0.01$ & $32.5 \%$ & $30.5 \%$ & $<0.01$
\end{tabular}




\begin{tabular}{lllllll} 
Multivessel Revascularization & $20.1 \%$ & $41.3 \%$ & $<0.01$ & $67.5 \%$ & $52.4 \%$ & $<0.01$ \\
Hypertension & $78.3 \%$ & $87.2 \%$ & $<0.01$ & $84.5 \%$ & $84.9 \%$ & 0.48 \\
Diabetes Mellitus & $37.5 \%$ & $49.7 \%$ & $<0.01$ & $46.7 \%$ & $46.1 \%$ & 0.47 \\
Hyperlipidemia & $68.9 \%$ & $76.8 \%$ & $<0.01$ & $73.3 \%$ & $74.2 \%$ & 0.05 \\
Chronic Kidney Disease & $16.5 \%$ & $20.8 \%$ & $<0.01$ & $21.5 \%$ & $20.6 \%$ & 0.16 \\
Congestive Heart Failure & $25.0 \%$ & $34.4 \%$ & $<0.01$ & $33.1 \%$ & $32.4 \%$ & 0.40 \\
Systolic Heart Failure & $16.2 \%$ & $23.1 \%$ & $<0.01$ & $21.6 \%$ & $21.3 \%$ & 0.66 \\
Coronary Artery Disease & $86.6 \%$ & $93.6 \%$ & $<0.01$ & $90.7 \%$ & $92.0 \%$ & $<0.01$ \\
Chronic Anemia & $12.9 \%$ & $21.2 \%$ & $<0.01$ & $19.7 \%$ & $18.6 \%$ & 0.09 \\
Atrial Fibrillation & $14.0 \%$ & $30.6 \%$ & $<0.01$ & $21.8 \%$ & $22.6 \%$ & 0.18 \\
Atrial Flutter & $1.7 \%$ & $4.0 \%$ & $<0.01$ & $2.9 \%$ & $3.0 \%$ & 0.80 \\
Long-term Anticoagulation & $5.0 \%$ & $4.7 \%$ & 0.16 & $5.4 \%$ & $4.9 \%$ & 0.23 \\
Aspirin & $21.8 \%$ & $26.3 \%$ & $<0.01$ & $25.2 \%$ & $25.4 \%$ & 0.78 \\
Abnormal Coagulation Profile & $0.3 \%$ & $0.6 \%$ & $<0.01$ & $0.4 \%$ & $0.5 \%$ & 0.19 \\
Peripheral Vascular Disease & $10.7 \%$ & $16.0 \%$ & $<0.01$ & $15.4 \%$ & $14.4 \%$ & 0.09 \\
Chronic Pulmonary Disease & $17.4 \%$ & $23.7 \%$ & $<0.01$ & $22.3 \%$ & $22.0 \%$ & 0.62 \\
Chronic Liver Disease & $1.8 \%$ & $3.1 \%$ & $<0.01$ & $2.5 \%$ & $2.6 \%$ & 0.78 \\
Smoking & $47.0 \%$ & $53.0 \%$ & $<0.01$ & $49.8 \%$ & $50.9 \%$ & 0.19 \\
Obesity & $19.1 \%$ & $29.4 \%$ & $<0.01$ & $25.1 \%$ & $25.3 \%$ & 0.81 \\
\hline
\end{tabular}

AMI: acute myocardial infarction, SD: standard deviation.

Table 2: Study endpoints before and after propensity matching.

\begin{tabular}{llll}
\hline & Before Propensity Matching & $\begin{array}{l}\text { Before Propensity Matching } \\
\text { SR }\end{array}$ & $\begin{array}{l}\text { Before Propensity Matching } \\
\text { P-value }\end{array}$ \\
Endpoint/Group & PR & 9,550 & - \\
Number of Patients & 35,989 & $2.2 \%$ & $<0.01$ \\
All-cause Mortality & $3.7 \%$ & 11.6 & $<0.01$ \\
Mean Length of Stay in Days & 4.3 & $1.8 \%$ & $<0.01$ \\
Stroke & $1.0 \%$ & $24.8 \%$ & $<0.01$ \\
Acute Kidney Injury & $14.9 \%$ & $47.1 \%$ & $<0.01$ \\
Bleeding & $4.3 \%$ & $18.5 \%$ & $<0.01$ \\
Blood Transfusion & $2.9 \%$ & $19.8 \%$ & $<0.01$ \\
Acute Respiratory Failure & $10.7 \%$ & $229,917 \$$ & $<0.01$ \\
Total Hospital Charges & $120,590 \$$ & & \\
\hline
\end{tabular}

PR: percutaneous revascularization, SR: surgical revascularization.

Figure 1: Flowchart demonstrating data extraction process. 


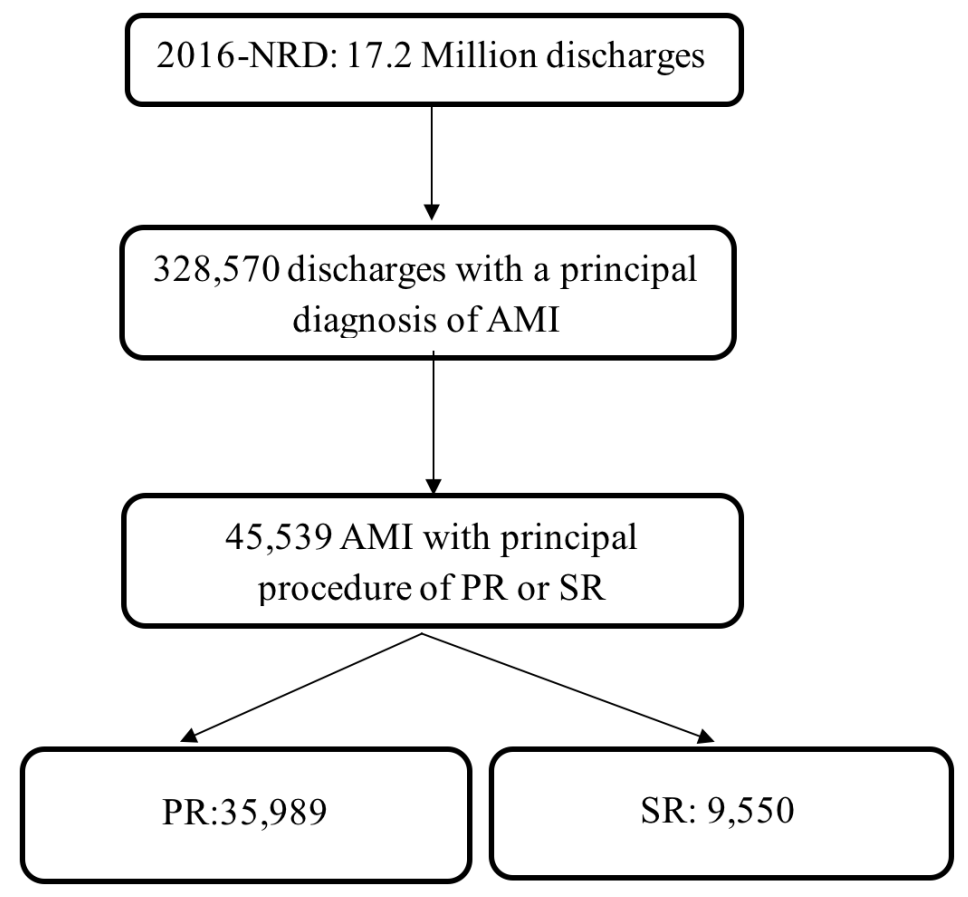

AMI: acute myocardial infarction, NRD: Nationwide Readmissions Data, PR: percutaneous revascularization, SR: surgical revascularization. 